\title{
LITERATURWISSENSCHAFT
}

http://dx.doi.org/10.18778/2196-8403.2008.10

BRIGITTE SCHULTZE

\section{Übersetzung von fiktionaler und nichtfiktionaler Prosa. Untersucht an polnisch-deutschen und polnisch-englischen Translationsfällen}

\begin{abstract}
Specyficzne wymagania translatoryczne przy tłumaczeniu prozy fikcjonalnej z jednej i prozy niefikcjonalnej z drugiej strony łączą się z różnorodnym stanem obydwu form narracji: w narracji fikcjonalnej przede wszystkim trzeba zwracać uwagę na wybrany przez autora gatunek narracyjny, tendencje i kody danej epoki, może i styl autorski; w wypadku narracji „faktualnej“ (MARTINEZ / SCHEFFEL 2005) zaś, gatunek niefikcjonalny jak $\mathrm{i}-\mathrm{z}$ reguły celowy - na specjalną uwagę zasługuje sposób traktowania faktów. Obydwa wybrane dla analiz porównawczych przykłady powstały w latach 1950: prozę fikcjonalną reprezentuje krótkie opowiadanie MARKA HŁASKI Krzyż (1956), niefikcjonalną albo półfikcjonalną zaś klasyczny pamiętnik z Gulagu GusTAWA HERLINGA-GRUDZIŃSKIEGO, Inny świat (1951/1952).
\end{abstract}

Die spezifischen translatorischen Herausforderungen beim Übersetzen von fiktionaler Prosa auf der einen und nichtfiktionaler Prosa auf der anderen Seite hängen mit dem unterschiedlichen Status beider Formen der Narration zusammen: Beim fiktionalen Erzählen sind vor allem das vom Autor gewählte narrative Genre, Epochenströmungen bzw. -codes, vielleicht eine individuelle Ästhetik zu beachten; beim „faktualen“ Erzählen (MARTINEZ / SCHEFFEL 2005) ist die nichtfiktionale Textsorte sowie der - in der Regel absichtsvolle - Umgang des Autors mit dem Faktenmaterial besonders wichtig. Beide für vergleichende Analysen gewählten Beispiele sind in den 1950er Jahren entstanden: Für die fiktionale Prosa steht MAREK HŁASKos kurze Erzählung Krzyż (,Das Kreuz', 1956), für die nichtfiktionale bzw. halbfiktionale Prosa stehen GUSTAV HERLING-GRUDZIŃSKIs kanonische Memoiren aus dem Gulag, Inny świat (,Eine andere Welt', 1951/1952).

The specific translatory challenges of prose fiction, on the one hand, and nonfictional narrative, on the other hand, are connected with the different status of both these forms of narration: In prose fiction, first of all, the genre chosen by the author, the trends and 
Brigitte Schultze

codes of an epoch and, maybe, the author's individual aesthetics have to be taken into account; in „factual“ narration (MARTINEZ / SCHEFFEL 2005), however, the nonfictional genre and also the author's - as a rule deliberate - way of dealing with the facts tend to be especially important. Both examples chosen for a comparative analysis were written in the 1950ies: Fictional prose is represented by MAREK HŁASKO's short narrative Krzyz (,The Cross', 1956), nonfictional prose, half-fictional prose respectively, by GUSTAV HERLING-GRUDZIŃSKI's , classical ' memoirs of the Gulag, Inny świat (,A World Apart', 1951/1952).

\section{I.}

Die spezifischen translatorischen Herausforderungen im Umgang mit fiktionaler Prosa einerseits und nichtfiktionaler Prosa andererseits hängen zunächst mit dem unterschiedlichen Status beider Formen der Narration zusammen. Matias Martinez und Michael Scheffel stellen den phänomenologischen Unterschied in dieser Weise heraus:

1. Erzählt werden kann von realen oder erfundenen Vorgängen.

2. Erzählt werden kann im Rahmen von alltäglicher Rede oder aber im Rahmen von dichterischer Rede (MARTINEZ / SCHEFFEL 2005:10).

Sie unterscheiden zwischen ,fiktionalem“ und „faktualem“ Erzählen (MARTINEZ / SCHEFFEL 2005:9-19). Was der phänomenologische Unterschied für Translationsvorgänge bedeutet, sei hier ganz kurz an zwei Beobachtungsorten fiktionalen (literarischen) und nichtfiktionalen (faktualen) Erzählens und Übersetzens in Erinnerung gerufen: dem Dialog sowie Eigennamen.

Im Kontext fiktionaler Prosa sind die Redebeiträge erzählender und erzählter Figuren Bestandteil einer erfundenen Textwelt und somit Bestandteil eines umfassenden ästhetischen Deutungsangebots. Der einzelne Redebeitrag unterstützt die schrittweise Erstellung des Bildes einer fiktionalen Gestalt, trägt gegebenenfalls durch Leitgedanken, wiederkehrendes Wortmaterial und anderes mehr zur Kohärenzbildung, vielleicht auch zu einer von Offenheit, Widersprüchlichkeit und Unfestigkeit bestimmten Textwelt bei. Übersetzer und Übersetzungsforscher haben in jedem Fall nicht nur den einzelnen Redebeitrag oder eine einzelne Prosaszene, sondern die Figurenrede in der Längsachse des Textes zu beachten. Mit Blick auf die Längsachse des Textes kann es z.B. geboten sein, dass ein bedeutungshaltiges Wort, das von Lesern des Ausgangstextes unmittelbar erfasst und in seiner Tragweite verstanden werden kann, für Leser der Zielkultur nochmals eingebracht, vielleicht graphisch hervorgehoben wird. 
Im Kontext nichtfiktionaler Prosa, die sich auf tatsächliche Gespräche bezieht, geht es vor allem um die einzelne Episode bzw. Situation. Der Übersetzer hat sich, ähnlich dem Autor, um eine möglichst genaue Wiedergabe von Wortlaut, paralinguistischen Signalsetzungen, vielleicht Pausen, zu bemühen. Einen Wortwechsel gemeinsam mit vorangehenden und nachfolgenden Redebeiträgen im Hinblick auf Sinnangebote des gesamten Textes zu befragen und die Befunde solcher Befragungen translatorisch umzusetzen, zählt in der Regel nicht zu seinen Aufgaben. Sofern Dialoge oder Dialogfragmente Realienbezeichnungen oder anderes Material der Lebenswelt enthalten, ist selbstverständlich in aufeinanderfolgenden Gesprächswiedergaben auf einen einheitlichen übersetzerischen Umgang mit solchen ,Belegen' zu achten.

Bei dem Beobachtungsort ,Eigennamen', d.h. Vor- und Familiennamen, auch Vatersnamen und weiteren kulturspezifischen Bestandteilen von Eigennamen, können in fiktionalen Texten sowohl Namen der Lebenswelt als auch okkasionelle Wortbildungen vorkommen. Gemäß einem verbreiteten Verständnis von der Kulturtechnik literarischen Übersetzens, wie es sich insbesondere in der zweiten Hälfte des 20. Jhd.s herausgebildet hat, besteht für Übersetzer zunächst die Möglichkeit zum Direkttransfer: zu einer unveränderten Hineinnahme eines Eigennamens in den Zieltext. Eigennamen können jedoch auch adaptiert, d.h. an die Lautung der Zielsprache angepasst, gegebenenfalls auch durch Namen ersetzt werden, die in der Zielkultur ähnliche Kontextbedeutungen mit sich führen und ähnlich ,funktionieren“ wie im Ausgangstext. Okkasionelle Namenprägungen bieten die Möglichkeit zu einer verfahrensanalogen Wiedergabe der einzelnen Bestandteile des - vielleicht singulären - fiktionalen Namens.

Derartige Wahlmöglichkeiten, die im Falle fiktionaler Prosa durch die konkrete Form, den historischen Roman etwa, eingegrenzt sein können, stehen den Übersetzern nichtfiktionaler Prosa nicht zur Verfügung. Bei Familiennamen ist Direktübernahme der gebotene Transfermodus; bei Vornamen kann auch die zielkulturelle Entsprechung des Eigennamens gewählt werden. Sofern ein Übersetzer, z.B. unter Zensurbedingungen - somit aus Gründen des Persönlichkeitsschutzes -, in seiner Vorlage enthaltene Eigennamen durch Initialen oder auch Pseudonyme unkenntlich bzw. schwer identifizierbar macht, ist er in der Regel gehalten, einen solchen Eingriff im Text kenntlich zu machen - in einer Annotation, im Nachwort usw.

Unterschiede zwischen der Übersetzung fiktionaler und nichtfiktionaler Prosa ließen sich an einer Reihe weiterer Beobachtungsorte veranschaulichen. Von fundamentaler Bedeutung ist offensichtlich der Merkmalkomplex, der mit den 
einzelnen fiktionalen sowie nichtfiktionalen Genres oder auch Formen (Textsorten) zusammenhängt. Es geht also um Formen wie Kurzgeschichte, Novelle, Erzählung und Roman auf der einen und Autobiographie, Tagebuch, Erinnerungen bzw. Memoiren, Reisebeschreibung usw. auf der anderen Seite. Historisch-vergleichende Übersetzungsanalysen zeigen, dass Übersetzer nicht selten sowohl bei den Formen fiktionaler als auch bei denjenigen nichtfiktionaler Prosa Textmerkmale wie die Präzision sprachlichen Ausdrucks, ebenso Dichte und Ökonomie, auch Lücken in der Informationsvergabe, etwa das Fehlen von Verknüpfungen zwischen Sätzen und Absätzen, in ihrer spezifischen Bedeutung unterschätzen. Sofern ein solches Aufmerksamkeitsdefizit den übersetzerischen Umgang mit einer Kurzgeschichte betrifft, kann das bedeutungsbildende Potential der Gattungspoetik selbst geschwächt sein: Ökonomie der Informationsvergabe, Kürze und Verfahren der Verkürzung, Verdichtung und Verweisung, die Suggestivität von Details z. B., sind bekanntlich gattungsrelevant (z.B. DURZAK 1994). ${ }^{1}$ Wo ein Übersetzer dichten, ökonomi-

1 Es sei daran erinnert, dass das Polnische über eine Reihe von Möglichkeiten zu ökonomischem, dichtem und semantisch offenem sprachlichen Ausdruck verfügt, die der Gattungspoetik der Kurzgeschichte entgegenkommen. Das zeigt sich gerade beim Vergleich mit dem Deutschen: Sprachlicher Ökonomie dienen z. B. die Auslassung der Kopula jest in unmarkierter Rede und der weitgehende Verzicht auf das Personalpronomen beim Verb, auch der gerade in schriftlichen Äußerungen intensive Gebrauch von Partizipien; das Merkmal der Suggestivität erfüllt der grammatische Sinn von Aspekten und Aktionsarten usw. Während bei der Übersetzungsrichtung Deutsch-Polnisch Möglichkeiten zu einer Steigerung sprachlicher Ökonomie und grammatikgestützter semantischer Offenheit bestehen, kann bei der Übersetzungsrichtung Polnisch-Deutsch eine Expansion, d.h. eine $\mathrm{Zu}-$ nahme an Wortmaterial, aber auch eine Definierung und damit Schließung grammatisch begründeter Offenheitsstellen unvermeidlich sein. Ein exemplarisches Beispiel ist Zofia Nałkowskas Kurzgeschichte Przy torze kolejowym (,Am Bahndamm') aus dem Zyklus Medaliony (,Medaillons', z. B. in NAŁKOWSKA 1989:222227.) Hier sind Verkürzung, Verweisung und Verdichtung am gesamten Material zur Textgenerierung realisiert - von Absätzen im Umfang eines einzigen Satzes und sinngebenden Interpunktionszeichen bis zur Lexik. Durch die Verwendung der Kopula ist und den Gebrauch der Personalpronomina sowie die Auflösung von Partizipien ist bei der Übersetzung ins Deutsche Expansion unvermeidlich. In HENRYK BERESKas Übersetzung dieser Kurzgeschichte, Am Bahndamm, gedruckt in HAGENAU (1963:17-21, hier 17), gibt es außer solchen unumgänglichen Transferproblemen des gattungspoetischen Programms auch einzelne Reduktionen bei den Merkmalen der Suggestivität und Verweisung. Die markierte Kollokation obóz zniszczenia z. B. (HAGENAU 1963:222, Vertilgungslager - siehe vernichtet werden, z. B. von Insekten) wird wie die gebräuchliche unmarkierte Bezeichnung obóz zagłady wiedergegeben: Vernichtungslager. In diesem und ähnlichen Fällen 
schen Ausdruck, das Fehlen verknüpfender Konjunktionen u.a.m. in Texten nichtfiktionaler Prosa unbeachtet lässt, ist dies zumeist weniger als Eingriff in ein gattungspoetisches Programm denn als Eingriff in den sprachlichen Duktus des jeweiligen Verfassers nichtfiktionaler Prosa zu sehen. Während ein Aufmerksamkeitsdefizit gegenüber der Gattungspoetik der Kurzgeschichte - analoge Befunde können sich im Umgang mit der klassischen Novelle zeigen unmittelbar die Generierung von Textsinn betrifft, kann mangelnde Beachtung der sprachlichen Textur dieser oder jener Form nichtfiktionaler Prosa vor allem die gedanklich-sprachliche Qualität beeinträchtigen.

In diesem Beitrag sollen übersetzerische Herausforderungen und Lösungen an einem Beispiel fiktionaler und einem Beispiel nichtfiktionaler Prosa aus den 1950er Jahren vorgestellt werden: an MAREK HŁASKOs 1956 erschienener Erzählung Krzyż (,Das Kreuz') und GuSTAw HERLING-GRUDZIŃSKIs Erinnerungen bzw. ,Dokumentarbericht' Inny świat - zapiski sowieckie (,Eine andere Welt - sowjetische Aufzeichnungen'), einem nichtfiktionalen oder auch halbfiktionalen Text, der zunächst, 1952, in englischer Übersetzung, danach erst, 1953, in der Ausgangssprache erschienen ist (SZENFELD 1990:732). Für einen Übersetzungsvergleich stehen bei beiden Fallbeispielen zwei deutsche Übersetzungen zur Verfügung. Bei Herling-Grudzińskis Inny świat wird auch die Übersetzung ins Englische in den Vergleich einbezogen. Im Falle von Hłaskos Krzyż interessiert der übersetzerische Umgang mit der fiktionalen Form, einer Erzählung mit deutlichen Merkmalen der Kurzgeschichte, sowie der Epochenkontext und die Individualästhetik des Autors (SCHULTZE 2008). Im Falle von Inny świat geht es vorrangig um die Textsorte Memoiren in der vom Autor gewählten Realisierung. Zu den Beobachtungsorten der Übersetzungsanalyse zählt hier auch der Vergleich zwischen den beiden deutschen Fassungen und dem englischen Übersetzungstext. Während sich die Befunde zu Hłaskos Text auf eine vollständige Analyse stützen können, haben die Befunde zu Inny świat eher vorläufigen Charakter. Bei der Großform konnten selbstverständlich nur ausgewählte Textstellen verglichen werden. Im Folgenden wird zunächst das Hłasko-Beispiel (II), danach der Text von Herling-Grudziński (III) betrachtet. In einem knappen Ausblick werden schließlich einige translatorische Herausforderungen bei fiktionalen und nichtfiktionalen Texten aufgezeigt, die in den hier gewählten Textfällen nicht vorkommen (IV).

markierter Wortwahl, okkasioneller Wortbildungen usw., die zumeist als unmarkiertes sprachliches Material in den Zieltext eingebracht werden, muss in der Regel offen bleiben, ob die Minderung von Signalsetzungen, und damit von Deutungsangebot, auf den Übersetzer selbst oder auf Verlagslektoren zurückgeht. 
Brigitte Schultze

II.

Die in Hłaskos nur fünf Seiten umfassender Kurzgeschichte bzw. Kurzerzählung Krzyż (HŁASKO 1963:113-118) enthaltenen Herausforderungen an Übersetzer lassen sich nicht nur benennen, sondern auch im Hinblick auf die Kontexte ihrer Entstehung verdeutlichen. Gerade die frühe Prosa trägt die Merkmale einer Individualästhetik (SCHULTZE 2008), die bei weiteren jungen Autoren Nachahmer gefunden hat. Zu dem Bedingungsrahmen, in dem Hłaskos frühe Prosa entstanden ist, gehört vor allem die unmittelbare Lebenserfahrung eines Autodidakten in der Zeit des Tauwetters, somit der ,Współczesność'-Generation (MAKOWIECKI 1992:497), und der Einfluss des amerikanischen Behaviorismus (BUDRECKI 1992:95-100). Der hier interessierende Text zählt im engeren Sinne zur Literatur der ,Pokolenie 1956“ (JAROSIŃSKI 1992:831). Die gedanklichen und ästhetischen Positionen des Sozialistischen Realismus bewusst ablehnend, gestanden diese Autoren einander individuelle Schreibweisen zu. An die Stelle ideologisch verordneter Schönfärberei trat eine ungeschminkte Wirklichkeitsdarstellung - bis hin zu einem bewussten Bruch mit traditionellen ästhetischen Verfahren (,„́wiadomy antyestetyzm“). Neben peripheren Lebensräumen wie der Warschauer Vorstadt war auch die Provinz - mit ihren tradierten, von marxistischem Denken unberührten Denkhaltungen - ein bevorzugter Darbietungsgegenstand (JAROSIŃSKI 1992:831).

Der Einfluss des amerikanischen Behaviorismus kommt weniger in der Wahl des dargestellten Lebensraums als im Umgang mit narrativen Verfahren zur Geltung. Sofern dieser Einfluss konsequent umgesetzt wird, sind Einblicke in das Bewusstsein erzählter Figuren - als Gedankenbericht, erlebte Rede oder innerer Monolog - unzulässig. Als Indiz für innere Befindlichkeit oder inneres Erleben wird äußeres Verhalten minutiös dargestellt. Der Narrator bleibt als unsichtbarer, nicht dramatisierter Erzähler im Hintergrund. Die Bedeutungsbildung muss somit von einem aktiven Leser geleistet werden. Mit Blick darauf, dass ein erheblicher Teil der ideologisch bestimmten Narrativik des Sozialistischen Realismus auf passive Leser eingestellt ist, liegt hier ein ästhetisches Gegenprogramm vor. Hłasko setzt die Vorgaben des Behaviorismus allerdings insofern nur begrenzt um, als er durchaus Einblicke in Bewusstseinsvorgänge zulässt. Die Art und Weise, wie dies geschieht, ist dabei bereits bedeutungsbildend. Sinngebend sind überdies eine straffe Erzählerrede mit rhythmisch organisierten Wiederholungsmustern und Gesprächsdarstellungen, die unmittelbar der Lebenswelt entnommen sein könnten. In ihnen sieht Marek Zybura das zentrale Element von Hłaskos Individualästhetik: „Knappe, komprimierte Dialoge (Hłaskos größte erzähltechnische Stärke) und ein nüch- 
terner Tatsachenstil ohne kommentierenden oder rhetorischen Ballast“" (ZYBURA:4).

Hier zeichnen sich bereits Herausforderungen für Übersetzer ab. Die von deutlichem künstlerischen Bauwillen gekennzeichnete Kurzerzählung Krzyż bestätigt und exemplifiziert den skizzierten Bedingungsrahmen von Hłaskos früher Prosa. Der dargestellte Vorgang findet innerhalb eines einzigen Tages im Vorfrühling statt. Gezeigt werden mehrere Stationen einer Gefängnisanlage sowie ein Fußweg vom Gefängnis zu einer Bahnstation. In zwei Erzählsequenzen, einer längeren und einer kürzeren, wird zunächst der Weg eines Gefangenen aus der Zelle in einen Empfangsraum, in dem er sich vor der Hinrichtung von seinen Eltern verabschieden darf, sodann der Weg der Eltern zur Bahnstation gezeigt. Die erste Erzählsequenz umfasst ihrerseits drei Mikrosequenzen, in denen das Verlassen der Zelle, der Gang des Gefangenen zum Empfangsraum und das letzte Gespräch mit den Eltern geboten sind. In meist knappen Gesprächsbeiträgen der wortarmen Eltern werden in der zweiten Erzählsequenz Bruchstücke des Mordgeschehens erkennbar: Während der Vater - nachdem er ein hinlänglich stabiles bäuerliches Anwesen geschaffen hatte - für seinen älteren Sohn eine Frau mit ,einem Stück Erde“ ausgesucht hatte, hatte der Sohn sich in eine „Bettlerin“ (,dziadówka“) verliebt und mit ihr zusammengelebt. Da im Verständnis des Vaters „ein Mensch“ „ohne Land“ nichts „,wert ist“, hatte jener seinem Sohn befohlen, die „Bettlerin“ umzubringen. Zur Genugtuung des Vaters hatte der Sohn gehorcht: „Musiał zrobić po mojej woli“ (,Er musste nach meinem Willen handeln“); „Synowskie posłuszeństwo miał““ (,Er hat den Gehorsam eines Sohnes [= kindlichen Gehorsam] gehabt"). Einen Vorschlag des Vaters zur Vertuschung der Mordtat hatte der - angesichts des Geschehens fassungslose - Sohn nicht angenommen bzw. nicht annehmen können (HŁASKO 1963:118).

Im Hinblick auf den extrem ökonomischen sprachlichen Ausdruck, bedeutungsbildende Wiederholungsstrukturen, das Verweispotential einzelner Wörter und anderes mehr bietet diese Erzählung das gattungspoetische Profil einer Kurzgeschichte. Ein Wortwechsel, mit dem der Sohn seine Eltern abzulenken sucht, ist allerdings ausführlicher dargeboten, als dies für das Deutungsangebot der Kurzgeschichte relevant erscheint; Ähnliches gilt für manche Details des Gefängnisalltags. In der ersten, umfangreicheren Erzählsequenz gibt es eine dichte sprachliche Textur, die einerseits auf verbale Ausdrücke des Wortfeldes siedzieć (,sitzen'), andererseits auf die Verben iść/chodzić (,gehen') sowie wstać (,aufstehen') gestützt ist. Das Wiederholungsmuster sitzen ist stellenweise durch den verbalen Ausdruck sich hinsetzen (siadać/usiqść) vertreten. 
Brigitte Schultze

Auf etwa vier Druckseiten finden sich mehr als 45 Strukturträger dieser Art. Das Wiederholungsmuster verweist nicht nur darauf, dass dem Bauernsohn seine an körperliche Bewegung gebundene Daseinsform genommen ist. Wichtiger noch ist eine weitere verweisende Funktion, die zum ,moment of truth', d. h. zu einer der gattungspoetischen Systemstellen der Kurzgeschichte, führt. ${ }^{2}$ Als der Vater seinen Sohn zum Abschied „mit dem Kreuze des Herrn“ ,segnet“ und befiehlt, jener solle „niederknien“, verweigert der Sohn diese Geste; stehend küsst er seinen Eltern die Hände (HŁASKO 1963:116). Im Sinne der Kurzgeschichte, die „,nicht psychologisiert“, sondern menschliche „Reaktionen" im Hinblick auf ihre Aussage befragt (DURZAK 1994:25), erkennt der Vater schlagartig, dass der Sohn ,zum ersten Mal“ „,nicht nach [seinem] Willen gehandelt" hat (HŁASKO 1963:117). Gattungstypisch ist die Suche nach dem ,moment of truth', dem Kern der Bedeutungsbildung, dem Leser im letzten Satz aufgetragen. Die Mutter des Todeskandidaten formuliert die Frage: „Ale dlaczego nie chciał uklęknać przed krzyżem?““ (,Aber warum wollte er nicht vor dem Kreuz niederknien?‘; HŁASKO 1963:118). Der mit dieser Frage auf die Signalstrukturen des Textes zurückverwiesene Rezipient ist dadurch in einer günstigeren Position als die erzählte Figur, dass Hłasko hier gezielt von den Vorgaben des Behaviorismus abweicht: Viermal wird ganz knapp Einblick in Bewusstseinsvorgänge des Todeskandidaten gegeben. Dabei geht es ausschließlich um dessen Körpergefühl: Nach langem Sitzen auf der Pritsche kann jener nur noch unter Schmerzen gehen, ,fürchtet sich davor aufzustehen“ und hat Angst vor dem „Schmerz“ beim Niederknien (HŁASKO 1963:115f.). Sowohl die dichte Textur zu dem Kontrastfeld sitzen/sich (hin)setzen: gehen/aufstehen als auch diese fast kryptischen Elemente des Gedankenberichts legen die Deutung nahe, dass der Todeskandidat mit der Weigerung niederzuknien (,beim Gedanken daran sah er Sterne vor seinen Augen“; HŁASKO 1963:116) zum einen weitere Schmerzen vermeidet, zum anderen einen Rest menschlicher Würde bewahrt.

Eine translatorische Bewältigung der markanten Textoberfläche mit ihrem gesamten Subtext hält nicht geringe Herausforderungen an Übersetzer bereit. Am Texteingang gibt es z.B. dieses auf das Verb siedzieć (,sitzen') gestützte Wiederholungsmuster: ,,ci, którzy siedzieli tu dłużej [...]. Człowiek siedzacy na pryczy [...]. Siedzacy na pryczy [...]. Siedzacy wstał“" (,diejenigen, die hier länger einsaßen [...]. Der auf der Pritsche sitzende Mensch (Mann) [...]. Der auf der Pritsche Sitzende [...]. Der Sitzende stand auf'; HŁasko 1963:113). Diese

2 Diese Systemstelle hat Hemingway, ein schriftstellerisches Vorbild von Hłasko, besonders herausgestellt (DURZAK 1994:52). 
vom Verb sitzen ausgehende schrittweise Reduzierung von Wortmaterial bis hin zur Einkreisung der bedeutungsbildenden Wörter sitzen - aufstehen lässt sich als Leserhilfe für die weitere Textlektüre sehen. Für Kenner mag sie ein impliziter Hinweis auf ein zentrales Verfahren der Kurzgeschichte sein. Ein Übersetzer wird hier an den Bauwillen des Werkproduzenten herangeführt. Eine wörtliche, d.h. ausgangstextnahe Übersetzung klänge noch markierter als im Ausgangstext. Auf das bedeutungsbildende Verb gehen wird gleichfalls durch eine Ballung bzw. durch ein Wiederholungsmuster aufmerksam gemacht. Dieses leitet das zweite Segment innerhalb der ersten Erzählsequenz ein:

Wyszli na korytarz. Strażnik zamknął drzwi. Poczęli iść korytarzem; więzień szedt przodem i swoje ogromne dłonie założył do tyłu. Naprzeciw nim szli dwaj więźniowie [...]. (HŁASKO 1963:113)

(Sie gingen hinaus auf den Korridor. Der Wächter schloss die Tür. Sie begannen, den Korridor entlangzugehen; der Häftling ging vornweg und seine riesigen Hände hatte er auf den Rücken gelegt. Ihnen entgegen gingen [= kamen] zwei Häftlinge $[\ldots])$.

Mit Ausnahme des verbalen Ausdrucks „Naprzeciw nim szli“ (,kamen ihnen entgegen') ließe sich das Wiederholungsmuster erhalten. Ähnliches gilt für eine Ballung des bedeutungsstiftenden Verbs am Übergang von der ersten zur zweiten Erzählsequenz. Hier wird das Fortgehen des Sohnes, das ein endgültiges Fortgehen aus dem Leben bedeutet - ,wyszedt“ (,er ging hinaus') - vom Fortgehen der Eltern, die das Gefängnis als freie Menschen verlassen, abgelöst:

[...] wyszedt razem ze strażnikiem. Starzy również wyszli; poszli przez dziedziniec [...]. Poczęli iść w kierunku dworca, skąd za dwie godziny odchodzit pociąg. Szli [...]. (HŁASKO 1963:116)

([...] er ging zusammen mit dem Wärter hinaus. Die Alten gingen gleichfalls hinaus; gingen über den Hof [...]. Sie begannen, in Richtung Bahnhof zu gehen, von wo in zwei Stunden der Zug abging. Sie gingen [...]).

Während die Wiedergabe der konkreten Bewegung des Gehens, ja selbst das ,Abgehen“ des Zuges im Zieltext ausgebracht werden können, entstehen dort Probleme, wo das Verbpaar iśćlchodzić und seine Ableitungen eine metaphorische Bedeutung haben. Der Vater leitet sein Eingeständnis, dass er seinem Sohn den Mord befohlen hat, mit den Worten ein: „tak wychodzi, że ja [...]“ (HŁASKO 1963:117) Die verbale Textur wäre, sofern der ,bewusste Antiästhetismus' ein translatorisches Ziel ist, bedacht, wenn man übersetzte: ,es geht darauf raus, dass ich [...]' Nur erfahrene, aufmerksame Übersetzer dürften diesen verbalen Ausdruck hier erhalten. Im Unterschied zu der Worttextur zum Sitzen/Sich (hin)setzen versus Gehen/Aufstehen, die sich weitgehend reprodu- 
zieren lässt, kann die präzise Verwendung des grammatischen Sinns der Verbalaspekte nicht angemessen an die Zielkultur weitervermittelt werden. Der Befehl des Vaters zum Niederknien vor dem Kreuz wird zunächst - unmarkiert - im perfektiven Aspekt, „Uklęknij“, danach - markiert - im imperfektiven Aspekt, „Klęknij, synu“, artikuliert (HŁASKO 1963:116). Hier könnten allenfalls translatorische Lösungen wie ,knie nieder - knie schon “ den Unterschied andeuten.

Hłaskos Kurzerzählung liegt in zwei deutschen Fassungen vor. Diese bilden das Übersetzungsgeschehen im deutschsprachigen Raum vor 1989 ab, das weitgehend ,zweispurig' war: Sehr oft standen ein Übersetzungstext aus der Bundesrepublik Deutschland oder auch aus Österreich und ein weiterer aus der DDR nebeneinander. In diesem Fall wurde die bundesrepublikanische Übersetzung von Hans Goerke (HŁASKO / GOERKE1967) ${ }^{3}$, diejenige aus der DDR von Hubert Schumann (HŁASKO / SCHUMANN 1979) erstellt. In Goerkes Übersetzung ist die auf die verbalen Ausdrücke gestützte Textur nicht mehr als Deutungsangebot zu erkennen. Dieses Wortmaterial ist auf ein Drittel gekürzt. Die Ballung des Verbs siedzieć am Texteingang ist z.B. deutlich abgebaut: ,[...] alle, die hier schon länger saßen [...]. Der Mann, der auf der Pritsche $s a \beta$ [...]. Der Mann auf der Pritsche [...]. Der Mann erhob sich von der Pritsche. (HŁASKO / GOERKE 1967:327) Das Partizip siedzqcy wird einmal in einen Relativsatz aufgelöst, sonst wird es fortgelassen. Während im Ausgangstext die beiden bedeutungsbildenden Verben siedzieć und wstać knapp und komprimiert aufeinanderfolgen, ,siedzacy wstat“, schafft der Übersetzer durch Hinzufügung von weiterem Wortmaterial (,von der Pritsche“) einen beschreibenden Satz, in dem Hłaskos sprachlicher Duktus aufgehoben ist. Von der Ballung des Verbpaares iśćlchodzić am Übergang zum zweiten Erzählsegment ist nur eine Nennung des Verbs gehen geblieben: „Sie verließen die Zelle; der Wärter schloß die Tür, und dann gingen sie den Korridor entlang: der Häftling voran, seine riesigen Hände auf dem Rücken verschränkt. Zwei andere Häftlinge [...] kamen ihnen entgegen." (HŁASKO/GOERKE 1967:327)

Ähnlich wie in diesem Textausschnitt verändert der Übersetzer auch sonst wiederholt die Interpunktion. Während im Ausgangstext zwei knappe Mitteilungen durch einen Punkt getrennt sind - ,Zamknął drzwi. Poczęli iśśc" (,schloß die Tür. Sie fingen an zu gehen ${ }^{6}$ ) - sind die Sätze hier durch ein Komma aneinandergereiht. Durch die Einfügung der Konjunktion und ist die Auflösung der ,harten' Satzgrenze des Ausgangstextes noch weiter getrieben.

3 Die Übersetzung lag bereits 1958 vor (HŁASKO 1958). 
Derartige Eingriffe in den Text gibt es häufiger: Syndetische Fügungen werden in asyndetische umgewandelt und umgekehrt, Partikeln und anderes Wortmaterial werden eingefügt. Ähnlich wie die Auflösung der Wiederholungsmuster aus den Verben iśćlchodzić bzw. wstać und siedzieć sowie siadać/usiaść führen auch diese übersetzerischen Veränderungen zu einer deutlichen Reduktion des ,bewusst antiästhetischen ' Schreibens der ,Generation 1956' - somit auch zum Abbau eines wesentlichen Merkmals von Hłaskos Individualästhetik. Beim Übergang von der ersten zur zweiten Erzählsequenz ist die Ballung des Verbpaares iść/chodzić z. B. auf die Hälfte verkürzt. Das geschieht durch stilistische Variation, die häufig mit einer Anhebung des stilistischen Niveaus verbunden ist. Aus der Formulierung ,poszli przez dziedziniec“(, sie gingen über den Hof'; HŁASKO 1963:116) wird „,ie überquerten den Gefängnishof“" (HŁASKO / GOERKE 1967:331). Hier wird überdies der ,Hof" bzw. das ,Hofgelände" übersetzerisch spezifiziert. Der Abbau des, unschönen' Stils zeigt sich z.B. auch, wenn der Satz , - No tak - rzekt ojciec. - Nie pora teraz rozmawiać" (,Na ja - sagte der Vater. - [Ist] nicht die Zeit, sich jetzt zu unterhalten“; HŁASKO 1963:116) wiedergegeben ist als: „,Nun ja“, versetzte der Vater. ,Jetzt ist nicht die Zeit, lange Unterhaltungen zu führen“" (HŁASKO / GOERKE 1967:330). ${ }^{4}$ Während die knappen Sprechansätze des spracharmen Vaters hier und an anderen Stellen erhalten sind, wird der knappe sprachliche Ausdruck in den längeren Mitteilungen wiederholt durch verschiedene Formen der Expansion wortreicher.

Die Tatsache, dass die bedeutungsstiftende Textur aus den Verben siedzieć sowie siadać/usiasśc einerseits und iść/chodzić sowie wstać andererseits durch Auslassungen und die Verwendung sinnverwandter Lexik weitgehend getilgt ist, hängt möglicherweise nicht allein mit einer Abneigung des Übersetzers gegen solche ,antiästhetischen" Wortballungen zusammen. Der Übersetzer sieht ein anderes als das oben skizzierte Deutungsangebot im Zentrum von Krzyż. Er benennt diesen Sinnkern in einem Untertitel, für den in den polnischen Hłasko-Ausgaben keine Anhaltspunkte gefunden werden konnten: „Ein Kommentar zum vierten Gebot“ (HŁASKO / GOERKE 1967). Es steht außer Frage, dass das vierte Gebot - „Du sollst deinen Vater und deine Mutter ehren,

4 Der Umgang mit den sich wiederholenden Verba dicendi - rzekt/powiedziat (, sagte er'), die hier stilbildend bzw. , codetypisch' verwendet sind, erfolgt ähnlich wie der Umgang mit dem verbalen Ausdruck he said in vielen englisch-deutschen und englisch-polnischen Übersetzungen: Das Wiederholungsmuster, das bei englischsprachigen Lesern gar nicht als solches wahrgenommen wird, wird durch synonymische Ausdrücke aufgehoben. 
Brigitte Schultze

auf das dirs wohlgehe und du lange lebest auf Erden“ - eine wichtige Teilmenge des Deutungsangebots ist: Der in einem geradezu archaischen Denken gefangene Bauer (daran hat der Kommunismus nichts geändert) folgt einem pervertierten Verständnis des vierten Gebots. Dieses Gebot ist noch um den Preis eines Verstoßes gegen das fünfte Gebot einzuhalten; ,kindlicher Gehorsam' macht den Sohn zum Mörder und bringt ihm den Tod. Wenn der Sohn und sei es, um sich weiteren (ihn demütigenden) physischen Schmerz zu ersparen - seinem Vater den Kniefall vor dem Kreuz verweigert, so distanziert er sich, zumindest indirekt, auch von dem pervertierten Verständnis des vierten Gebots und der gesamten Manipulation der christlichen Religion.

Um sein Textverständnis an deutschsprachige Leser weiterzugeben, wählt der Übersetzer Goerke drei Formen translatorischer Veränderung: 1. eine weitgehende Tilgung des Wiederholungsmusters sitzen/gehen, 2. die Hinzufügung eines Untertitels und 3. die Auslassung mehrerer Sätze. Fortgelassen ist der letzte Gedankenbericht, in dem die Furcht des Todeskandidaten vor Schmerzen und Augenflimmern beim Kniefall wiedergegeben ist. ${ }^{5}$ Eine so weitgehende translatorische ,Absicherung ' eines Textverständnisses ist, das zeigt die Erfahrung mit Übersetzungsanalysen zu fiktionaler Prosa, in der 2. Hälfte des 20. Jhd.s eher eine Ausnahme denn die Regel. ${ }^{6}$ So bleibt festzuhalten, dass die bereits 1958 (vgl. Anm. 3), somit in großer Nähe zur Entstehungszeit des Ausgangstextes, vorliegende erste deutsche Übersetzung von Krzyż in dreifacher Hinsicht von der polnischen Vorlage abweicht: 1. Der Zieltext exponiert eine Teilmenge des Deutungsangebots, die im Ausgangstext lediglich als komplementärer Ort der Sinngenerierung erscheint; 2. das - zwar nicht exemplarisch realisierte, jedoch hinlänglich erkennbare - bedeutungsbildende Inventar der Kurzgeschichte wird durch den hinzugefügten Untertitel sowie die Reduktion von Verfahren der Ökonomie, Verdichtung und Verweisung so weitgehend aufgehoben, dass die lockerer angelegte Form der Erzählung entsteht; 3. durch die Auflösung von Wiederholungsfiguren aus identischem Wortmaterial, die stilistische Anhebung einzelner Wörter und Wortgruppen, Eingriffe in die Interpunktion u.a.m. sind viele Signalsetzungen zu Hłaskos Individualästhetik, insbesondere auch die , antiästhetische Sprachgebärde " der ,Generation 1956“ unkenntlich gemacht. Dass Hłaskos Status als polnischer Kultautor nicht nur

5 Damit entfällt auch eine der beiden Nennungen des Imperativs von klękaćl klęknqúc, somit das Problem, den grammatischen Sinn der Verbalaspekte bedenken zu müssen.

6 Ein eigenständiges Problemfeld ergibt sich selbstverständlich dort, wo narrative Texte aus politischen Gründen in spezifischer Weise verändert werden mussten. 
mit den behandelten Themen und einer ungeschönten Darstellung der Lebenswelt, sondern auch mit einem ,antiästhetischen' sprachlichen Duktus verbunden ist, kann also von deutschsprachigen Lesern kaum oder gar nicht nachvollzogen werden.

Als Hubert Schumanns Übersetzung von Krzyż in der DDR erschien, 1979, war die Zeit des ,Tauwetters' längst Vergangenheit, waren in Polen andere Schriftsteller (z.B. Iredyński) vorübergehend in den Status von Kultautoren gelangt. Schumann verzichtet auf die Hinzufügung eines rezeptionssteuernden Untertitels. Er erhält die vier kurzen Einblicke in Bewusstseinsvorgänge des Häftlings. Rezipienten können somit die verschiedenen Teilmengen des Deutungsangebots - die mit Schmerz und Demütigung verbundenen Körpererfahrungen des Bauernsohnes, die Pervertierung der christlichen Gebote - aufnehmen. Die Textoberfläche mit ihren Wiederholungsstrukturen zum Sitzen/ Hinsetzen, Gehen/Aufstehen, der konjunktionslosen Zusammenfügung von Haupt- und Nebensätzen u. a. m. ist auch hier teilweise übersetzerisch getilgt. Es gibt jedoch mehr Textspuren als in der ersten deutschen Übersetzung. Die einleitende, nur um den Preis erheblicher Störungen zu reproduzierende Ballung des Verbs siedzieć (,sitzen') ist z.B. so wiedergegeben: ,[...] alle, die hier länger saßen [...]. Der Mann auf der Pritsche [...]. Der Mann auf der Pritsche [...]. Der Mann stand auf und reckte sich“ (HŁASKo / SchumanN 1979:152). Hier ist ein bewusster Umgang mit der translatorischen Herausforderung zu erkennen. Die Wendung „Der Mann auf der Pritsche“, die dann nochmals dem Ausgangstext verfahrensanalog folgend - verkürzt wiederkehrt, ersetzt die Ballung des Verbs. Das Verb gehen ist in der Längsachse des Textes häufiger ausgebracht als in Goerkes Übersetzung: „Wir gehen nicht über den Hof [...]. Wir gehen gleich nach unten [...]. Auf dem Korridor ging der Häftling voran" (HŁASKO / SCHUMANN 1979:152). Weitgehend reproduziert ist z.B. auch das Verbum dicendi rzekt (,sagte er'; HŁASKO 1963:117; HŁASKO / SCHUMANN 1979:156f.). Auf der anderen Seite werden aber auch in dieser Übersetzung asyndetische Fügungen in syndetische verwandelt, wird wiederkehrendes Wortmaterial variiert, häufig zugleich stilistisch angehoben. Die Rückfrage des Gefängniswärters „Co pan - żarty gra?“ (etwa: ,Was denn - Sie machen Witze?‘; HŁASKO 1963:114) wird zu: „Sie wollen mich wohl auf die Schippe nehmen?" (HŁASKo / SCHUMANN 1979:153) Die Bestätigung der auffallend spracharmen Bäuerin, dass ihr Sohn „gut“ gewesen sei - „Dobry, dobry“ (HŁASKO 1963:118), wird zu: „Ein guter Kerl, eine Seele von Mensch“ (HŁASKO / SCHUMANN 1979:157). Mit solchen translatorischen Eingriffen wird nicht nur Hłaskos ,größte erzähltechnische Stärke', die Reproduktion knapper menschlicher Rede, unbeachtet gelassen; es werden zugleich die Sprechpor- 
träts der erzählten Figuren verändert. Es bleibt festzuhalten, dass die in der früheren DDR entstandene Übersetzung der Kurzerzählung Krzyż deutschsprachige Leser insgesamt angemessener mit Hłaskos kurzer Prosa vertraut machen kann als die in der Bundesrepublik Deutschland entstandene und in mehreren Editionen verbreitete Wiedergabe von Hans Goerke. ${ }^{7}$ Aus der historischen Distanz gewinnen beide Übersetzungen eine weitere Facette: Eine konsequente ausgangstextnahe Reproduktion des ,antiästhetischen " Sprachduktus der ,Generation 1956' würde im 21. Jhd. wahrscheinlich als veraltet, manieristisch - befremdlich - erscheinen.

III.

Bei nichtfiktionaler Prosa der zweiten Hälfte des 20. Jhd.s sind die Unterschiede zwischen Ausgangs- und Zieltext zumeist weniger gravierend als in dem hier betrachteten Beispiel fiktionaler Prosa. Das gilt selbstverständlich insbesondere für ,innereuropäische " Translationsfälle, weniger für Fallbeispiele, bei denen große sprachliche und kulturelle Andersheit zu bedenken ist. Bei Formen wie Tagebuch, Reisebeschreibung, Autobiographie, Erinnerung u. a.m. ist die Menge translatorischer Abweichungen, z.B. auffälliger Verschiebungen in den mitgeteilten Inhalten, in der Regel recht begrenzt. Manchmal nehmen die Abweichungen dort zu, wo die nichtfiktionalen Textsorten zur fiktionalen Prosa hin geöffnet sind oder wo von vornherein Mischformen mit einem hohen Anteil ästhetischer Verfahren, etwa auch Codemischung und Codewechsel, gegeben sind. Im Falle von Herling-Grudzińskis Inny świat (,Eine andere Welt') geht es, wie gesagt, um die Form der Memoiren (,Pamiętnik“; LEMANN 2006:507f.) mit einer sehr spezifischen Öffnung zur fiktionalen Prosa. ${ }^{8}$ Das gestalterische Moment hängt mit der erinnerten „Welt“ zusammen: dem sowjetischen Lagersystem Gulag, das Herling-Grudziński nach mehrmonatiger Gefängnishaft - etwa anderthalb Jahre lang in Ercevo erleben musste, bis er 1941 in Verbindung mit einer Amnestie für polnische Gefangene und dem Aufbau der Armee von General Anders nach Italien ent-

7 Dieser Tendenzbefund einer größeren ,Professionalität‘ im Umgang mit fiktionalen Texten hat sich mehrfach bestätigt, insbesondere in Übersetzungstexten, die in den 1960er und 1970er Jahren entstanden sind. Möglicherweise zeigen sich hier die Erfolge der sorgfältigen Ausbildung von Übersetzern in der DDR.

8 Die Teilhabe an fiktionalem wie auch an nichtfiktionalem Schreiben kommt in Olga SzENFELDs Werkartikel (1990:732) darin zum Ausdruck, dass sie von einem ,romanartigen“ Dokumentarbericht spricht. 
kam. Im Sinne von Scheffel und Martinez bezieht sich Herling-Grudziński auf „reale Vorgänge“. „Alltägliche Rede“ kann diesem Erleben jedoch nicht genügen; es fehlt ein Beschreibungsvokabular. Mit Blick auf die Notwendigkeit der Sprachfindung in einem solchen Fall extremer Erfahrungen gilt ganz besonders diese Feststellung von RICHARD RORTY (1989:6): „The world does not speak. Only we do. The world can, once we have programmed ourselves with a language, cause us to hold beliefs. But it cannot propose a language for us to speak." An dieser Stelle können das von Herling-Grudziński gefundene Beschreibungsvokabular und die Elemente literarischer Gestaltung ${ }^{9}$ nicht vorab erschlossen werden. Dazu bedürfte es einer eigenständigen Forschung. ${ }^{10}$

Auch wenn weder das Beschreibungsvokabular noch die im Text enthaltenen einzelnen Linien gedanklicher Auseinandersetzung aufgezeigt werden können, lassen sich einige Grundmerkmale dieser halbfiktionalen Prosa nennen: Es geht um eine überaus faktenreiche, präzise, um Objektivität ringende Darstellung, der funktionale Wechsel von Bericht, Beschreibung und zitierter Rede ist unmittelbar nachzuvollziehen; der Verfasser der Memoiren bleibt weitgehend hinter dem Bericht unsichtbar; er meldet sich nur dort in der Ich-Form zu Wort, wo er ein persönliches Urteil artikuliert oder von persönlichem Erleben spricht. ${ }^{11}$ Die übersetzerischen Herausforderungen liegen ohne Frage zunächst in der bedachten Wortwahl und darin, dass Herling-Grudziński die Möglichkeiten des Polnischen zu knappem, präzisem Ausdruck umfassend nutzt; auch die Reihenfolge der Informationsvergabe innerhalb jedes Satzes kann den Übersetzer fordern, ebenso einzelne Vokabeln aus dem Kontext der Welt des Gulag sowie der Politik und Verwaltung der Sowjetunion.

9 Auf diese Seite von Inny świat hat Alfred Gall in einem im Juli 2007 an der Universität Mainz gehaltenen Vortrag zu Recht hingewiesen: ,, Auf unmenschlicher Erde'. Die Auseinandersetzung mit dem stalinistischen Terror in der polnischen Literatur".

10 Es sei daran erinnert, dass die 1951 abgeschlossenen und 1953 erstmals im Druck erschienenen Memoiren Inny świat der Kernphase von Herling-Grudzińskis literarischem Schaffen, zu der die Erzählungen der 1960er Jahre und der 1971 edierte Dziennik pisany nocq (,Tagebuch in der Nacht geschrieben') gehören, um mehr als zehn Jahre vorausgehen. Bei Inny świat ist eine gewisse Sonderstellung zu bedenken. EWA BIEŃKOWSKA (2002) bezieht diesen Text denn auch nicht in ihre Werkgeschichte ein.

11 BiEŃKOWSKA (2002:118) weist darauf hin, dass der Autor selbst häufig auf die Modellfunktion der alten Chroniken hingewiesen habe: deren ,unpersönliche Kälte, Objektivität [...]“. 
An dieser Stelle sollen nur die Wiedergabe des Titels und des Inhaltsverzeichnisses sowie ausgewählte Textstellen aus den beiden Kapiteln „Praca“ (,Arbeit") und „Ręka w ogniu“ (,Die Hand im Feuer") interessieren. In beiden Fällen handelt es sich um Auszüge aus dem ersten der beiden Teile der Memoiren. Sowohl der Titel als auch die Überschriften der einzelnen Kapitel bzw. Unterkapitel lassen bereits eine Öffnung der nichtfiktionalen Form der Memoiren zur fiktionalen Prosa erkennen. Der Titel Inny świat (,[Eine] Andere Welt $^{\circ}$ ) bezieht sich auf Dostoevskijs 1862 erstmals vollständig gedruckte Memoiren Zapiski iz Mertvogo doma (,Aufzeichnungen aus [einem] Totenhaus'). Im ersten Absatz des I. Kapitels, „Mertvyj dom“ (, Totenhaus“), heißt es: „,Tut byl svoj osobyj mir, ni na čto bolee ne pochožij [...].“ (,Hier war eine eigene, besondere [abgesonderte, separate] Welt, eine nichts Anderem weiter ähnliche [...]'; DostoevskiJ 1972:9). Diese Textstelle liefert in Herling-Grudzińskis Memoiren sowohl den Titel als auch das Motto: „Tu otwierał się inny, odrębny świat, do niczego niepodobny [...].“ (,Hier eröffnete sich eine andere, besondere [eigenartige, separate] Welt, eine nichts ähnliche'; HERLING-GRUDZIŃSKI 1989: [5]). Während der russische Prätext das „Eigene, Besondere“ der Lagerwelt hervorhebt (,svoj osobyj [...] svoi osobye [...] svoi [...] svoi [...]"), wird in dem polnischen Motto das „Andere, Besondere“ betont: „inny, odrębny [...] inne [...] inne [...] inne“. Dieser Unterschied ist insofern deutungsrelevant, als Herling-Grudziński zu einem anderen Verständnis der Lagerwelt gelangt als Dostoevskij in seinen Aufzeichnungen aus einem Totenhaus. ${ }^{12}$ Die translatorische Abweichung zwischen dem russischen „svoi“ (,eigene“) und dem polnischen ,inny“ (,andere“) sollte somit bei der Wiedergabe des Titels Inny świat bedacht sein. Weitere fundamentale Unterschiede zwischen Dostoevskijs Memoiren und denjenigen Herling-Grudzińskis seien wenigstens angesprochen: Während bei Dostoevskij die Dokumentation sehr deutlich hinter der fiktionalen Ausgestaltung zurücktritt (GERIGK 1989:824f.), steht bei Herling-Grudziński die Vorstellung von Fakten im Vordergrund; während Dostoevskij einen fiktiven Adressaten schafft, den er in die Lagerwelt mitnimmt, wird die Aufmerksamkeit der Rezipienten in Inny świat ausschließlich auf die existentielle Extremsituation der Lagerwelt gelenkt.

In den ausschnitthaften Übersetzungsvergleich sollen drei Übersetzungstexte einbezogen werden: 1 . die 1952, somit vor dem Ausgangstext, erstmals publizierte englische Wiedergabe von Joseph Marek - A World Apart - (HERLING / MAREK 1974), 2. die nach der englischen Übersetzung von Hansjürgen Wille erstellte erste deutsche Übersetzung (HERLING / WILLE 1953) und die am Aus-

12 Diesen Befund hat Gall (vgl. Anm. 9) mit besonderem Nachdruck versehen. 
gangstext orientierte Überarbeitung dieser Erstübersetzung durch Nina Kozlowski (HERLING / WILLE / KOZLOWSKI 2000). Es soll nicht darum gehen, diese drei Übersetzungstexte systematisch nacheinander mit der Vorlage zu vergleichen. Hier interessieren vielmehr zum einen Beispiele für Nähe zum Ausgangstext, zum anderen auffällige Abweichungen.

$\mathrm{Zu}$ den Auffälligkeiten gehört zunächst der übersetzerische Umgang mit dem Titel. Da im Englischen eine Direktübersetzung von Inny świat entfällt - mit ,A Different World“ wäre die Andersartigkeit nicht hinlänglich ausgedrückt, das Adjektiv „other-worldly“ meint eine weltferne, , abgehobene“ Haltung kann das nachgestellte Adverb „apart“" (,getrennt', ,abseits', ,separat') dem Ausgangstext ohne Frage am besten gerecht werden. Hiermit wird, substituierend, auf die semantische Ausfüllung von „odrębny“ zurückgegriffen. ${ }^{13} \mathrm{Ob}-$ wohl die deutsche Sprache, anders als die englische, eine Wiedergabe des Titels mit ,Eine andere Welt ' zulässt, tragen beide deutsche Fassungen den Titel Welt ohne Erbarmen. Falls Wille bei der Erstellung seiner Erstübersetzung allein den englischen Titel $A$ World Apart kannte, hätte er mit einer translatorischen Lösung wie ,Eine Welt für sich' den Zusammenhang mit dem Ausgangstext wahren können. Der von ihm eingeführte und dann beibehaltene Titel Welt ohne Erbarmen führt nun sowohl von der englischen Übersetzung als auch vom Ausgangstext fort. Die offene Aussage der Vorlage wird durch eine sinnmindernde Interpretation ersetzt. Diese Titelwiedergabe ist, zumindest teilweise, eine ,freie Neuformulierung “114, wie sie im internationalen Übersetzungsgeschehen eher die Ausnahme als die Regel ist. Mit Welt ohne Erbarmen sind mehrere deutliche Abweichungen geschaffen: 1. Sowohl die semantische Offenheit des Titels als auch der Hinweis auf die fundamentale Andersheit der Welt des Gulag sind aufgehoben; 2. getilgt ist die "Intertitularität“ (NORD 2004:912), d.h. die in Herling-Grudzińskis Memoiren und in der englischen Übersetzung erkennbare Beziehung zu Dostoevskijs Aufzeichnungen aus einem Totenhaus; 3. dadurch, dass die Neuformulierung ein emotionales Element exponiert, entsteht ein Widerspruch zu Herling-Grudzińskis um „Kühle“ bzw. „Kälte“ und „Objektivität“ bemühtem Bericht.

13 Die englische Wiedergabe des Mottos beginnt so: „Here there is a world apart, unlike everything else, with laws of its own, its own manners and customs [...].“ (HERLING / MAREK 1974: Titelblatt).

14 Christiane NoRD (2004:911) stellt - allerdings im Hinblick auf ein von ihr gewähltes Corpus literarischer Texte - fest, dass bei etwa 10 Prozent der Titel vom Verfahren „freier Neuformulierung“ Gebrauch gemacht werde. 
Brigitte Schultze

Ähnlich dem Titel ist auch das Inhaltsverzeichnis ein besonders sensibler Ort des übersetzerischen Transfers. Dass Kapitelüberschriften derartig übersetzungsrelevant sind, scheint dabei in Texten des 20. Jhd.s eher für nichtfiktionale und halbfiktionale als für fiktionale Prosa kennzeichnend zu sein. Bei Inny świat kommt der Gestaltungswille des Autors Herling-Grudziński darin zum Ausdruck, dass die Gefängnisstationen außerhalb des Gulag, der Tradition nichtfiktionaler Memoiren folgend, als raum-zeitliches Nacheinander geboten sind - Witebsk - Leningrad - Wołogda - (HERLING-GRUDZIŃSKI 1989:[328]), der dokumentarische Bericht zur, Anderen Welt' selbst jedoch systematischen Akzenten folgt: „Praca“ (,Arbeit'), „Dom swidanij“ (,Haus der Begegnungen“ [ $=$ des Wiedersehens]). Die übersetzerischen Wiedergaben dieser Kapitel und Unterkapitel sind insgesamt auffallend eng an der jeweiligen Vorlage orientiert. Hier gibt es fast keine Abweichungen. $\mathrm{Zu}$ den wenigen Auffälligkeiten gehört, dass das Unterkapitel „Ochłap“ (,Abfall“, ,Übrigbleibsel', ,Rest“, ,Brocken') explizierend wiedergegeben ist als "Thrown to the wolves" (HERLING / MAREK 1974:IX) bzw. „Den Wölfen zum Fraß hingeworfen“ (HERLING / WILLE 1953:277; HERLING / WILLE / KOZLOWSKI 2000:337). Während der Ausgangstext das Lebensende des einstigen Folterers Gorcew, der nun den Gefangenen zur Rache überlassen ist, mit der Chiffre ,Brocken', , Abfall' anzeigt, verdeutlicht die englische und nach ihr die deutsche Übersetzung den dargestellten Vorgang. Dabei wird auf den letzten Satz des Unterkapitels zurückgegriffen. Es geht um den Kommentar eines Gesprächspartners, eines Ingenieurs, dem Herling-Grudziński von dem Vorfall berichtet hatte. Im Unterschied zur fiktionalen Prosa interessiert hier nicht das Sprachporträt der erzählten Figur, sondern allein der Inhalt und die Art, wie er in Worte gefasst ist. Dieser eine ,zur Dokumentation“ zitierte Satz endet so: , ,...] teraz rzuca się lwy na pożarcie niewolnikom“ (, [...] jetzt wirft man die Löwen den Sklaven zum Fraß vor'; HERLING-GRUDZIŃSKI 1989:73). Das Bild der Christenverfolgung in Rom ist mit einem anderen Raubtier besetzt, dem Wolf. Während der deutsche Bandtitel Welt ohne Erbarmen im Wortsinn Bedeutung verstellt, lässt sich hier translatorischer Gewinn sehen: Es entsteht zusätzliche Intertitularität zu einem weiteren nichtfiktionalen Text der zweiten Hälfte des 20. Jhd.s, Nadežda Mandel'štams 1970 erschienener, vielzitierter Autobiographie Das Jahrhundert der Wölfe (KEMP 1990:8).

Neben diesem Beispiel für übersetzerischen Gewinn enthält das Inhaltsverzeichnis auch einen Fall von unvermeidbarem Verlust an Bedeutungsbildung. Dieser rührt von der Mehrsprachigkeit der Kapitelüberschriften her. Die Mehrsprachigkeit geht in jedem Fall dort verloren, wo nichtpolnisches Vokabular mit der Sprache eines Übersetzungstextes zusammenfällt. Die Kapitelüber- 
schrift „Drei Kameraden“ ist zwar in der englischen, nicht aber in der deutschen Übersetzung als fremdsprachliches Element mit nicht geringer semantischer Aufladung zu identifizieren. Dem russischen Terminus „Dom Svidanij“ (,Haus der Begegnungen') geht sowohl die Fremdsprachigkeit als auch der im Ausgangstext durch Anführungszeichen und Kursivdruck hervorgehobene - Charakter eines Terminus verloren, wenn die englische Kapitelüberschrift „The House of Meetings“ (HERLING / MAREK 1974:[VII]) lautet. Diese translatorische Option ist nachvollziehbar: Polnische Rezipienten können den russischen Terminus verstehen, englische hingegen nicht.

Im Folgenden sei ganz knapp der übersetzerische Umgang mit Herling-Grudzińskis präziser, extrem dichter Sprache nachvollzogen. Dafür, dass die englische Übersetzung dem Ohr eines Muttersprachlers als gelungen erscheint, können Aussagen des Philosophen Bertrand Russel gelten. Sie sind sowohl den englischen als auch den deutschen Textausgaben als Vorwort vorangestellt:

Of the many books [...] relating the experiences of victims in Soviet prisons and labour camps [...] A World Apart is the most impressive and the best written. He [Herling] possesses in a very rare degree the power of simple and vivid description, and it is impossible to question his sincerity at any point. (HERLING / MAREK 1974:IX)

Das erste Beispiel stammt aus dem Unterkapitel „Stalins Mörder“. Hier geht es um den Einsatz der Nachtblinden bei der Waldarbeit. Im Ausgangstext heißt es: „Kurzych ślepców nie posyłano naturalnie nigdy do robót, które przeciągały się do późnej nocy“ (,Nachtblinde wurden natürlich niemals zu Arbeiten geschickt, die sich in die späte Nacht hinzogen'; HERLING-GRUDZIŃSKI 1989: 74). Die englische Übersetzung lautet: „The night-blind were naturally never assigned to work which sometimes lasted far into the night" (HERLING / MAREK 1974:51). Die Übersetzung enthält zwei Abweichungen: Das unmarkierte Wort posytano (,geschickt') wird durch den mehr, verfahrenstechnischen ' Ausdruck assigned (,eingesetzt', ,beordert') ersetzt; das Adverb sometimes ist, sofern Marek nicht eine andere Vorlage hatte, ein hinzugefügtes Element. In den gleichlautenden deutschen Übersetzungen fehlt dieses Wort manchmal. Die Vorlage lässt das englische Verb to assign erkennen: „Die Nachtblinden wurden natürlich nie bei einer Arbeit eingesetzt, die bis spät in die Nacht dauerte." (Herling / WILle 1953:60; HeRling / WiLLE / KoZLOWSKI 2000:72) Ungeachtet der Abweichungen machen alle drei Übersetzungstexte die gleiche inhaltliche Aussage. Manche geringfügige Abweichungen, auf die hier nicht eingegangen werden soll, rühren offensichtlich daher, dass Herling-Grudziński selbst seinen Text nochmals geringfügig überarbeitet 
hat. Doch gibt es, gerade in den deutschen Wiedergaben, auch einige Abweichungen, die das sprachliche Profil des Gesamttextes tangieren. Die Beispiele schließen an den zuvor gesetzten Satz an. Im Ausgangstext folgt diese Mitteilung: „W naszej brygadzie tragarzy nie było ich nigdy [...]“ (,In unserer Trägerbrigade gab es sie nie [...]'; HERLING-GRUDZIŃSKI 1989:74). Gemeint sind die Nachtblinden. Dem englischen Erstübersetzer hat offensichtlich eine etwas andere Fassung vorgelegen. Sein Text lautet: „They were never found in the porter's brigade [...]“ (HERLING / MAREK 1974:51). Entsprechend lauten beide deutschen Übersetzungen: „Darum gehörte auch keiner von ihnen zur Trägerbrigade [...]“" (Herling / WILle 1953:60; HerLING / WILle / KozlowSKI 2000:72; Hervorhebung - B.S.). Das Adverb darum ist ein Zusatz der deutschen Fassung. Hier zeigt sich ein Trendbefund: In beiden deutschen Übersetzungen gibt es wiederholt Adverbien und Konjunktionen, die kausale Zusammenhänge - über den Ausgangstext hinaus - verstärken. Wenige Sätze weiter lautet z.B. ein Satzanfang der englischen Wiedergabe: „He won the bet, but it cost him his life when, a few month later [...]“ (HERLING / MAREK 1974:51). Beide deutsche Übersetzungen geben diesen Satzeingang so wieder: „Er gewann die Wette, aber sie kostete ihn das Leben. Einige Monate danach nämlich, als [...]“ (HERLING / WILle 1953:60; HerLing / WiLle / KOZlowsKI 2000:73). ${ }^{15}$ Auch wenn solche Einfügungen, für die es weder in der englischen

15 Die syntaktische Vereinfachung, d. h. die Auflösung des Kettensatzes in zwei Sätze, kann wiederum mit unterschiedlichen Vorlagen zusammenhängen. - Die Unterschiede zwischen der ersten und der zweiten deutschen Übersetzung sind, soweit Stichproben dies erkennen lassen, insgesamt geringfügig. Es zeichnet sich ab, dass Kozlowski bei ihrer Durchsicht von Willes Erstübersetzung - wie gesagt, nach der englischen Fassung - eine ältere polnische Version des Textes verwendet hat. Dies sei wiederum an dem systematischen Abschnitt über die Nachtblinden veranschaulicht. Ein Absatz endet in der englischen Übersetzung (HERLING / MAREK 1974:51) folgendermaßen: ,,,The old man can't keep it up“, the Finn Rusto Karinen said to me with his strange accent.“ (,Der alte Mann kann nicht mithalten [Schritt halten], sagte der Finne Rusto Karinen mit seinem fremden [seltsamen, merkwürdigen] Akzent zu mir.') Die beiden deutschen Übersetzungen (HERLING / WiLle 1953:60; Herling / WiLle / KoZlowsKi 2000:73) lauten hier: „,Der alte Mann kommt nicht mit uns mit", sagte der Finne Rusto Karinen mit seinem fremdländischen Akzent zu mir." Die Wiedergabe von strange mit ,fremdländisch', die Kozlowski von Wille übernimmt, ist als translatorische Option insofern zu hinterfragen, als auch das Russische des Erzählers Herling-Grudziński nicht als ,inländisch', , authentisch“ usw. bezeichnet werden kann. In den neueren polnischen Ausgaben (HERLING-GRUDZIŃSKI 1989:75), die Kozlowski nicht eingesehen zu haben scheint, lautet diese Textstelle: „-- Starik - powiedział mi nawet na ucho ze śmiesznym akcentem rosyjskim Fin Rusto Karinen - nie 
noch in der polnischen Vorlage einen Anhaltspunkt gibt, nicht durchgängig in die deutschen Zieltexte eingebracht sind, beeinträchtigen sie die Rezeption des Textes: Die ,Kühle' und ,Strenge des sprachlichen Duktus' wird abgebaut; überdies ist - zumindest implizit - ein Rezipient in die Memoiren eingeschrieben, der ein Mehr an Leserführung braucht. Neben punktuell verstärkter Leserführung der beschriebenen Art gibt es auch punktuell vermehrte Rezipienteninformation. Sie liegt dort vor, wo in den Übersetzungstext selbst, d.h. in den Haupttext, Erläuterungen eingefügt sind, die im polnischen Ausgangstext entbehrlich erscheinen. Es handelt sich z.B. um erläuternde Hinweise zu Realienbezeichnungen. Dass derartige Informationen direkt in den Haupttext eingehen, ist dabei durchaus kennzeichnend für nichtfiktionale Prosa; in fiktionaler Prosa ist ein solches Verfahren weniger akzeptiert. ${ }^{16}$ In dem Kapitel „Ręka w ogniu“ (,Die Hand im Feuer'), das dem als exemplarisch zu sehenden Schicksal des Häftlings Michail Alekseevič Kostylev ${ }^{17}$ gewidmet ist, heißt es z.B.: „Należał wprawdzie najpierw do komsomołu, a potem do partii [...].“ (,Er gehörte zwar zunächst zum Komsomol und danach zur Partei [...]. 'HERLINGGRUDZIŃSKI 1989:98) Rezipienten der beiden deutschen Übersetzungen erhalten hier die Information: „Er gehörte zwar dem Komsomol (der kommunistischen Jugendorganisation) und später der Partei an [...].“ (HERLING / WILLE 1953:79; HERLING / WILLE / KOZLOWSKI 2000:96) Während bei polnischen Lesern Vertrautheit mit dem Terminus Komsomol vorausgesetzt ist, wird bei deutschsprachigen Lesern, vor allem denjenigen der Bundesrepublik vor 1989, Informationsbedarf angenommen. Die im Übersetzungstext entstehende Expansion ist akzeptiert. Da solche Einfügungen nur sparsam verwendet werden, ist der straffe sprachliche Duktus der Memoiren davon nicht beeinträchtigt. $\mathrm{Ob}$

może nadążyćc (,- Der Alte - sagte mir der Finne Rusto Karinen mit [seinem] komischen russischen Akzent sogar ins Ohr - kann nicht mitkommen. ') Hier ist durch größere Genauigkeit im polnischen Text eine missverständliche Übersetzung vermieden. Mit Blick auf ,Objektivität' als einem der Leitkonzepte von Herling-Grudzińskis Bericht entfällt z.B. eine Wiedergabe des Adjektivs śmieszny mit ,lächerlich'. Abweichungen der hier vorgestellten Art führen selbstverständlich nicht zu einem veränderten Bild der, Anderen Welt .

16 In fiktionaler Prosa sind solche Verstehenshilfen eher in Anmerkungen, manchmal auch in Annotationen im Textanhang gegeben.

17 Es ist durchaus möglich, dass hier - in Übereinstimmung mit dem dokumentarischen Charakter von Inny świat - der authentische Name des Häftlings gegeben wird. Die englische Übersetzung (HERLING / MAREK 1974:[VI]) und mehrere der polnischen Ausgaben von Inny świat enthalten den Hinweis des Autors, dass „einige Namen“ aus Gründen ,kluger Vorsicht“" geändert worden seien. 
sich an dieser oder jener Stelle dennoch sinnwidrige Abweichungen finden, ließe sich nur durch vollständige Analysen der Übersetzungstexte ermitteln.

\section{IV.}

Die vergleichende Übersetzungsanalyse von je einem Beispiel fiktionaler und nicht- bzw. halbfiktionaler Prosa aus den 1950er Jahren erlaubt eine vorsichtige Formulierung von Tendenzbefunden: Sowohl bei den beiden deutschen Übersetzungen von Hłaskos Kurzerzählung Krzyż als auch bei den beiden deutschen Fassungen von Herling-Grudzińskis Memoiren Inny świat gibt es Verfahren translatorischer Verstehenssicherung. Bei der fiktionalen Prosa wirken sich die gewählten Verfahren sehr viel mehr auf die Bedeutungsbildung aus als bei der nichtfiktionalen Prosa. Auch wenn Schumanns in der DDR entstandene Übersetzung von Krzyż sowohl Hłaskos Individualästhetik als auch den gattungspoetischen Verfahren der Kurzgeschichte mehr gerecht wird als Goerkes Übersetzung aus der früheren Bundesrepublik Deutschland, ist in beiden Wiedergaben der spezifische, ,antiästhetische" sprachliche Duktus des frühen Hłasko teilweise abgebaut. Über mögliche Ursachen für diese translatorischen Abweichungen soll hier nicht spekuliert werden. Man darf davon ausgehen, dass damit eine Akzeptanz Hłaskos bei deutschen Lesern erleichtert worden ist. In der historischen Perspektive lässt sich in einem gewissen Abbau individualästhetischer und zeitbedingter stilistischer Markiertheit manchmal ein Vorteil sehen: Derartige translatorische Lösungen sind gegebenenfalls länger ,ästhetisch akzeptabel ${ }^{\star}$, d.h. sie veralten weniger schnell. Sofern einmal ein Nachdruck vorgesehen ist, sollte in jedem Fall auf die Übersetzung von Schumann zurückgegriffen werden. Hier gibt es selbstverständlich auch eine andere, bessere Option: eine völlige Neuübersetzung, die sich im sprachlichen Duktus an Hemingways Prosa bzw. an guten Hemingway-Übersetzungen ins Deutsche orientiert.

Für die hier betrachtete nicht- bzw. halbfiktionale Prosa gilt, dass die verschiedenen Leserhilfen, denen weitere hinzugefügt werden könnten, die Textaussage nicht grundlegend verändern. Die meisten Verstehenshilfen sind von der Art, dass nun ein möglichst großer Rezipientenkreis mit einem weitgehend ,störungsfreien ' Lektüreangebot erreicht wird. Der Preis dafür ist eine gewisse Reduktion der Ökonomie, Strenge und sprachlichen Disziplin des Ausgangstextes. So bleibt festzuhalten, dass beide Translationsfälle nur in begrenztem Umfang eine Reproduktion der ästhetischen Markierungen wagen. Dieser Befund wiederholt sich in vielen Übersetzungen mit Deutsch als Zielsprache. Mit der Reduktion von Signalsetzungen ästhetischer Störung, vielleicht sogar Ver- 
störung, geht auch etwas vom Eigenwert ästhetischer Kommunikation verloren. Zugleich sind, so darf angenommen werden, , lektürebereite Leser' hinzugewonnen. Soweit erkennbar, stehen gerade polnisch-deutsche Übersetzungen des 20. Jhd.s im Zeichen des hier beschriebenen Trendbefunds.

Die wenigen Belegstellen aus Mareks englischer Erstübersetzung von Inny świat gestatten selbstverständlich noch keine Formulierung von Tendenzbefunden zum Transfer nichtfiktionaler polnischer Prosa ins Englische. Vor dem Hintergrund langjähriger Übersetzungsvergleiche lässt sich dennoch so viel festhalten: Mareks überaus sorgfältige Wiedergabe der ersten Fassung von Inny świat, in welcher die sprachliche Ökonomie des Ausgangstextes erhalten ist, entspricht nicht wenigen polnisch-englischen Übersetzungstexten. Dabei ist zu bedenken, dass das Übersetzungsaufkommen deutlich geringer ist als das deutsch-polnische. ${ }^{18}$ Bei den quantitativ begrenzten polnisch-englischen Übersetzungen wird in vielen Fällen gewagt, ein Mehr an ästhetischer Markiertheit, z.B. an verstörenden ästhetischen Lücken, an die Zielkultur weiterzugeben. ${ }^{19}$ So entsteht der Eindruck, dass hier eher für einen begrenzten Kreis kompetenter Rezipienten übersetzt wird als im deutschsprachigen Raum, in dem die polnische Literatur breiteren Leserkreisen vorgestellt werden soll. Für eine solche Beobachtung lassen sich selbstverständlich auch translatorische Gegenbeweise finden.

\section{Literatur}

Bereska, Henryk (1963): Am Bahndamm. In: Hagenau, Gerda (ed.]: Polen erzählt. Zweiundzwanzig Erzählungen. Frankfurt (M.), 17-21.

BieŃKowska, Ewa (2002): Pisarz i los. O twórczości Gustawa Herlinga-Grudzińskiego. [Der Schriftsteller und sein Schicksal. Über das Werk Gustaw Herling-Grudzińskis]. Warszawa.

BrodzKa, AlinA et al. (eds.): Stownik literatury polskiej XX wieku. [Lexikon der polnischen Literatur des 20. Jahrhunderts]. Wrocław.

BUDRECKI, LECH (1992): Behawioryzm. [Behaviorismus]. In: BRODZKA, 95-100.

18 Auch Hłaskos Prosa ist weniger ins Englische übersetzt worden als ins Deutsche. Eine englische Wiedergabe von Krzyż war nicht zu ermitteln.

19 Vgl. die Analysebefunde zu Adam Czerniawskis Übersetzung von Różewicz’ kanonischem Drama Kartoteka (,The card index") ins Englische (Schultze / MAKARCZYK-SCHUSTER 2008) sowie zu M. J. Krynskis und R. A. Maguires englischer Übersetzung von Szymborskas Kurzgedicht Próba (,Attempt‘) (SCHultze 2008). 
Brigitte Schultze

Dostoevskij, Fedor Michajloviè (1972): Zapiski iz Mertvogo doma. Leningrad (=Polnoe sobranie soèinenij 4).

DURZAK, MANFRED (1989 / $\left.{ }^{2} 1994\right)$ : Die Kunst der Kurzgeschichte. Zur Theorie und Geschichte der deutschen Kurzgeschichte. München.

GerigK, Horst-JÜRgen (1989): Zapiski iz Mertvogo doma. In: Kindlers Neues Literatur Lexikon. Bd. 4. München, 824f.

HeRling, Gustaw (1953): Welt ohne Erbarmen. Aus dem Polnischen von Hansjürgen Wille. Köln.

- (1974): A World Apart. Aus dem Polnischen von Joseph Marek. Westport (Connecticut).

- (2000): Welt ohne Erbarmen. Aus dem Englischen von Hansjürgen Wille und nach der polnischen Originalausgabe vollständig revidiert von Nina Kozlowski. München/Wien.

HERLING-GRUDZIŃSKI, GUSTAW (1989): Inny świat - zapiski sowieckie. Warszawa.

HŁASKO, MAREK (1958): Der achte Tag der Woche und andere Erzählungen. Aus dem Polnischen von Maryla Reifenberg, Hans Goerke und Vera Cerny. Köln/Berlin, 351-357.

- (1963): Krzyż. In: Opowiadania. [Erzählungen]. Paryż, 113-118.

- (1967): Das Kreuz. Ein Kommentar zum vierten Gebot. Aus dem Polnischen von Hans Goerke. In: Dedecius, KARL (ed.): Polnische Prosa des 20. Jahrhunderts. München, 327-332.

- (1979): Das Kreuz. Deutsch von Hubert Schumann. In: HŁasko, MareK: Hafen der Sehnsucht. Berlin, 152-157.

JAROSIŃSKI, ZBIGNIEW (1992): Pokolenia literackie. [Literarische Generationen]. In: BRODZKA, 825-833.

Kemp, Friedhelm (1990): Nadežda Jakovlevna Mandel’štam. Vospominanija. [Das Jahrhundert der Wölfe]. In: Kindlers Neues Literatur Lexikon. Bd. 11. München, 8.

KryszTOFIAK, MARIa (ed.) (2008): Ästhetik und Kulturwandel in der Übersetzung. Frankfurt (M.)/Berlin/Bern u. a. (=Posener Beiträge zur Germanistik 19).

Lemann, Natalia (2006): Pamiętnik. [Tagebuch]. In: GAZDA, GRZEGORZ / TYNECKAMAKOwSKA, SŁowinia (eds.): Stownik rodzajów i gatunków literackich. Kraków, 506-509.

MAKOWIECKI, ANDRZEJ Z. (1992): Krytyka literacka. [Literarische Kritik]. In: BRODZKA, 489-498.

Martinez, Matias / Scheffel, Michael (1999 / $\left.{ }^{6} 2005\right)$ : Einführung in die Erzähltheorie. München.

NAŁKOWSKA, ZoFIA (1989): Opowiadania. Charaktery dawne i ostatnie. Ściany świata. Medaliony. [Erzählungen. Alte und jüngste Charaktere. Die Wände der Welt. Medaillons]. Warszawa. 
Übersetzung von fiktionaler und nichtfiktionaler Prosa

NORD, CHRISTIANE (2004): Die Übersetzung von Titeln, Kapiteln und Überschriften in literarischen Texten. In: KITTEL, HARALD et al. (eds.): Übersetzung, Translation, Traduction. Ein internationales Handbuch zur Übersetzungsforschung. Berlin/New York (=Handbücher zur Sprach- und Kommunikationswissenschaft 26.1), 908-914.

RorTy, Richard (1989): Contingency, Irony and Solidarity. Cambridge.

SCHULTZE, BRIGITTE (2008): Individualästhetik als Beobachtungsort literarischen Übersetzens. In: KRYSZTOFIAK, 9-37.

Schultze, BrigitTe / MAKARCZYK-Schuster, Ewa (2008): Theorie und Praxis des Dramenübersetzens. Ein Werkstattbericht. In: KRYSZTOFIAK, 255-270.

SzEnfeld, Olga (1990): Gustaw Herling-Grudziński. A World Apart. In: Kindlers Neues Literatur Lexikon. Bd. 7. München, 732f.

ZyBuRA, MAREK: Marek Hłasko. In: Kritisches Lexikon zur fremdsprachigen Gegenwartsliteratur - KLFG-. 40. Nlg., 1-D/2. 